\title{
ON MEASURE AND OTHER PROPERTIES OF A HAMEL BASIS
}

\author{
R. B. DARST ${ }^{1}$
}

F. B. Jones has established [2] several interesting measure-related properties of a Hamel basis. In particular, he established the existence of a Hamel basis which contains a nonempty perfect set (and, hence, supports a nontrivial Borel measure) and commented on the plausibility of the notion that a Hamel basis should be in some sense "thick." Our purpose is to complement Jones' results by showing, subject to the continuum hypothesis which we assume throughout, that there exists a Hamel basis which intersects each first category set in, at most, a countable set and, hence, has universal measure zero (cf. [1]). It then follows, for instance, that the sum $E+F=\{e+f ; e \in E$, $f \in F\}$ of a universal null set $E$ and a universal null set $F$ need not be a universal null set and, moreover, an iterate $E^{n}$ of $E$ need not be Lebesgue measurable (cf. [2]).

In order to fulfill our purpose it suffices to establish the following theorem. (We wish to acknowledge collaboration with R. E. Zink on problems related to the content of this note.)

THEOREM. There exists a Hamel basis $H$ which intersects each perfect nowhere dense set in, at most, a countable set.

Before proceeding to a proof of the theorem we wish to state the following lemma which we shall have occasion to use.

LEMMA. If $Q$ is a first category subset of $(0,1]$ and $x$ is a point of $(0,1)$, then there exists a point $y$ of $(0, x)$ such that $x+y \in(x, 1)$ and neither $y$ nor $x+y$ is an element of $Q$.

Proof of Theorem. Let $\Omega$ denote the first uncountable ordinal and let $\left\{P_{\alpha}\right\}_{\alpha<\Omega}$ and $\left\{x_{\alpha}\right\}_{\alpha<\Omega}, x_{1}=1$, be well orderings of the perfect nowhere dense subsets of $(0,1]$ and the points of $(0,1]$. We shall define $H=\mathrm{U}_{\alpha<\Omega} H_{\alpha}$ inductively as follows. Let $H_{1}=\{1\}, R_{1}=\varnothing$. Suppose $1<\alpha<\Omega$ and, for $1 \leqq \beta<\alpha, H_{\beta}$ and $R_{\beta}$ satisfy:

(1) $H_{\beta}$ is, at most, countable.

(2) The elements of $H_{\beta}$ are linearly independent over the rationals.

(3) $R_{\beta}$ is a subset of the linear span $H_{\beta}^{\mathrm{L}}$ of $H_{\beta}$.

(4) $H_{\beta} \cap R_{\beta}=\varnothing$.

Received by the editors March 22, 1964.

1 Supported by National Science Foundation Grant GP03515. 
(5) $\left(H_{\beta} \cup R_{\beta}\right) \supset \bigcup_{\gamma \leq \beta}\left\{x_{\gamma}\right\}$.

(6) $R_{\beta} \subset U_{\gamma \leq \beta}\left\{x_{\gamma}\right\}$.

(7) $H_{\gamma} \subset H_{\beta}, R_{\gamma} \subset R_{\beta}, \gamma<\beta$.

(8) If $x \in H_{\beta}-H_{\gamma}$, then $x \in P_{\gamma}(\beta>\gamma)$.

In order to simplify what follows, let $K_{\alpha}=\bigcup_{\beta<\alpha} H_{\beta}, S_{\alpha}=\bigcup_{\beta<\alpha} R_{\beta}$, and $T_{\alpha}=\cup_{\beta<\alpha} P_{\beta}$. We now consider cases:

(a) If $x_{\alpha} \in K_{\alpha}$, let $H_{\alpha}=K_{\alpha}$ and $R_{\alpha}=S_{\alpha}$.

(b) If $x_{\alpha} \in K_{\alpha}^{\mathrm{L}}-K_{\alpha}$, let $H_{\alpha}=K_{\alpha}$ and $R_{\alpha}=S_{\alpha} \cup\left\{x_{\alpha}\right\}$.

(c) If $x_{\alpha} \notin K_{\alpha}^{\mathrm{L}} \cup T_{\alpha}$, let $H_{\alpha}=K_{\alpha} \cup\left\{x_{\alpha}\right\}$ and $R_{\alpha}=S_{\alpha}$.

(d) If $x_{\alpha} \in T_{\alpha}-K_{\alpha}^{\mathrm{L}}$ and there exists a rational number $r$ such that $r x_{\alpha} \in(0,1)-T_{\alpha}$, let $\lambda$ be the least index such that $x_{\lambda}$ is a rational multiple of $x_{\alpha}$ in $(0,1)-T_{\alpha}$ and then let $H_{\alpha}=K_{\alpha} \cup\left\{x_{\lambda}\right\}$ and $R_{\alpha}$ $=S_{\alpha} \cup\left\{x_{\alpha}\right\}$.

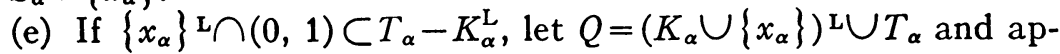
ply the lemma to obtain the least index $\lambda$ such that $x_{\alpha}$ and $x_{\lambda}$ play the role of $x$ and $y$ of the lemma. Then let $H_{\alpha}=K_{\alpha} \cup\left\{x_{\lambda}\right\} \cup\left\{x_{\lambda}+x_{\alpha}\right\}$ and $R_{\alpha}=T_{\alpha} \cup\left\{x_{\alpha}\right\}$.

\section{BIBLIOGRAPHY}

1. R. B. Darst, A remark on universal sigma integrability, Proc. Amer. Math. Soc. 14 (1963), 861-862.

2. F. B. Jones, Measure and other properties of a Hamel basis, Bull. Amer. Math. Soc. 48 (1942), 472-481.

Purdue University 